\title{
Topological nonrealization results via the Goodwillie tower approach to iterated loopspace homology
}

\author{
NICHOLAS KUHN
}

\begin{abstract}
We prove a strengthened version of a theorem of Lionel Schwartz [17] that says that certain modules over the Steenrod algebra cannot be the mod 2 cohomology of a space. What is most interesting is our method, which replaces his iterated use of the Eilenberg-Moore spectral sequence by a single use of the spectral sequence converging to $H^{*}\left(\Omega^{n} X ; \mathbb{Z} / 2\right)$ obtained from the Goodwillie tower for $\Sigma^{\infty} \Omega^{n} X$. Much of the paper develops basic properties of this spectral sequence.
\end{abstract}

$55 \mathrm{~S} 10 ; 55 \mathrm{~T} 20,55 \mathrm{~S} 12$

\section{Introduction and main results}

In this article, I prove some constraints on the mod 2 cohomology of spaces in which Steenrod squares "jump over gaps". Said otherwise, for certain unstable $\mathcal{A}$-modules $M$ with operations jumping over gaps, there are no spaces $X$ having $\widetilde{H}^{*}(X ; \mathbb{Z} / 2) \simeq M$. Here $\mathcal{A}$ is the mod 2 Steenrod algebra, and a module $M$ is unstable if $\mathrm{Sq}^{s} x=0$ for all $x \in M$ and $s>|x|$.

In [17], Lionel Schwartz established an interesting theorem of this type. The structure of his proof went as follows. Given $M \in \mathcal{U}$ of a specified sort, one wishes to show that no space $X$ exists with $H^{*}(X ; \mathbb{Z} / 2) \simeq M$. Assuming the existence of such an $X$, he showed that there could be no unstable algebra structure compatible with the $\mathcal{A}$-module structure on $H^{*}\left(\Omega^{n} X ; \mathbb{Z} / 2\right)$, where $n$ is a number determined by $M$. Here we recall that an unstable algebra satisfies both the Cartan formula, $\operatorname{Sq}^{k}(x \cup y)=$ $\sum_{i+j=k} \mathrm{Sq}^{i} x \cup \mathrm{Sq}^{j} y$, and the Restriction axiom, $\mathrm{Sq}^{|x|} x=x^{2}$.

The essence of his argument is elegant, and makes clever use of the product structure in the Eilenberg-Moore Spectral Sequence for computing $H^{*}(\Omega X ; \mathbb{Z} / 2)$, in conjunction with the structural form of the Adem relation for $\mathrm{Sq}^{2^{k}} \mathrm{Sq}^{2^{k}}$. Less elegant is his $n$-fold iterated use of the EMSS, necessitating inductive bookkeeping arguments.

The main point of our paper here is to give a new proof of Lionel's theorem, keeping the "fun" parts of his proof, but just using a single spectral sequence: the one associated to the Goodwillie tower for the functor sending a space $X$ to the spectrum $\Sigma^{\infty} \Omega^{n} X$. Our proof ultimately 
- yields a strengthened version of Lionel's theorem,

- gives some geometric meaning to what is being studied (the second stage of the tower), and

- illustrates the efficacy of using Goodwillie towers to study classical questions.

To state our main theorem we need to describe some unstable $\mathcal{A}$-modules. Inside $H^{*}(B \mathbb{Z} / 2 ; \mathbb{Z} / 2)=\mathbb{Z} / 2[t]$, the $\mathcal{A}$-module $\mathcal{A} \cdot t$ has basis $\left\{t, t^{2}, t^{4}, \ldots\right\}$, with $\mathrm{Sq}^{2^{k}} t^{2^{k}}=t^{2^{k+1}}$. For $k<l$, let $\Phi(k, l) \in \mathcal{U}$ be the subquotient with basis $\left\{t^{2^{k}}, \ldots, t^{2^{l}}\right\}$.

The modules we will be concerned with have the form $M \otimes \Phi(k, k+2)$, where $M$ is an unstable $\mathcal{A}$-module concentrated in degrees between $m$ and $l$. If $2^{k}>m-l$, then this unstable module is three copies of $M$, with $\mathrm{Sq}^{2^{k}}$ sending the first copy isomorphically to the second, and $\mathrm{Sq}^{2^{k+1}}$ sending the second copy to the third.

In formulae, the $\mathcal{A}$-module structure is described as follows: given $x \in M$ and $0 \leq s \leq m-l, \mathrm{Sq}^{s}\left(x \otimes t^{2^{i}}\right)=\mathrm{Sq}^{s}(x) \otimes t^{2^{i}}, \mathrm{Sq}^{s+2^{k}}\left(x \otimes t^{2^{k}}\right)=\mathrm{Sq}^{s}(x) \otimes t^{2^{k+1}}$, and $\mathrm{Sq}^{s+2^{k+1}}\left(x \otimes t^{2^{k+1}}\right)=\mathrm{Sq}^{s}(x) \otimes t^{2^{k+2}}$.

In pictures, $M \otimes \Phi(k, k+2)$ looks like

\begin{tabular}{|l|l|l|l|l}
\multicolumn{2}{c|}{$\mathrm{Sq}^{2^{k}}$} & $\mathrm{Sq}^{2^{k+1}}$ \\
\hline$M_{0}$ & $\sim$ & $M_{1}$ & $\sim$ & $M_{2}$ \\
\hline
\end{tabular}

where $M_{i}$ is the $2^{k+i}$-th suspension of $M$.

Our main theorem goes as follows.

Theorem 1.1 Let $M$ be an unstable $\mathcal{A}$-module concentrated in degrees between $l$ and $m$, such that its desuspension $\Sigma^{-1} M$ is not unstable. Suppose there exists a space $X$ such that $\tilde{H}^{*}(X ; \mathbb{Z} / 2) \simeq \Sigma^{n} M \otimes \Phi(k, k+2)$.

(a) If $n=0$, then $2^{k} \leq m-l$ must hold.

(b) If $n>0$, then $2^{k} \leq 4 m-2 l+2 n$ must hold. If, in addition, cup products are trivial in $\widetilde{H}^{*}(X ; \mathbb{Z} / 2)$, then $2^{k} \leq 4 m-2 l+2 n-2$ must hold.

By contrast, Schwartz' theorem [17, Theorem 0.2] just says that, for all $n, 2^{k} \leq$ $12(m+n)$ must hold. If, in addition, cup products are trivial in $\widetilde{H}^{*}(X ; \mathbb{Z} / 2)$, then $2^{k} \leq 12(m+n-1)$ must hold.

We note that the first statement in part (b) of the theorem (and also in Schwartz' theorem as just presented) is implied immediately by the second statement: if $X$ realizes $\Sigma^{n} M \otimes \Phi(k, k+2)$, then $\Sigma X$ realizes $\Sigma^{n+1} M \otimes \Phi(k, k+2)$, and cup products will be trivial in $\widetilde{H}^{*}(\Sigma X ; \mathbb{Z} / 2)$. 
Example 1.2 The theorem appears to be reasonably delicate; we consider what it says if $M=\mathbb{Z} / 2$, so that $l=m=0$.

When $n=0$, part (a) tells us the obvious fact that $\Phi(k, k+2)$ can't be realized for all $k \geq 0$.

When $n=1$, part (b) tells us that $\widetilde{H}^{*}(X ; \mathbb{Z} / 2) \simeq \Sigma \Phi(k, k+2)$ only if $k=0$ and the cohomology ring satisfies Poincaré duality in dimension 5. This does in fact happen, when $X=S U(3) / S O(3)$.

When $n=2$, part (b) tells us that $\tilde{H}^{*}(X ; \mathbb{Z} / 2) \simeq \Sigma^{2} \Phi(k, k+2)$ only if $k \leq 1$. In Section 6, we will look a bit more carefully at the proof of part (b) in this case, and we will show that $\tilde{H}^{*}(X ; \mathbb{Z} / 2) \simeq \Sigma^{2} \Phi(1,3)$ only if the cohomology ring satisfies Poincaré duality in dimension 10. This does in fact happen: an elegant construction of a smooth 10-dimensional manifold $M$ with this cohomology was given by E Floyd in [8, Section 3]. (Floyd also observes that the unoriented bordism classes of $S U(3) / S O$ (3) and $M$ are the only nonzero bordism classes represented by spaces with exactly 4 cells.)

Remarks 1.3 (a) The most well-known result of "mind the gap" type is due to JF Adams [1], and applies to spectra as well as spaces: if $k \geq 4, x \in H^{d}(X ; \mathbb{Z} / 2)$, and $H^{d+i}(X ; \mathbb{Z} / 2)=0$ for $0 \leq i \leq 2^{k}-2^{k-2}$, then $\mathrm{Sq}^{2^{k}} x$ must be in the image of $\mathrm{Sq}^{1}$.

(b) My interest in such questions goes back to my 1994 study [13] of spaces $X$ having $H^{*}(X ; \mathbb{Z} / 2)$ finitely generated over the mod 2 Steenrod algebra $\mathcal{A}$. Using Adams' theorem, I proved that, under the extra hypothesis that $\mathrm{Sq}^{1}$ acted trivially in high degrees, $H^{*}(X ; \mathbb{Z} / 2)$ would then have to be a finite dimensional $\mathbb{Z} / 2$-vector space. Furthermore, without the extra $\mathrm{Sq}^{1}$ hypothesis, the conjecture that this would still be true was reduced to various questions about the nonrealizability of various sorts of unstable $\mathcal{A}$-modules having operations jumping over gaps. In response to my paper, Lionel formulated and proved his theorem, as it suffices to prove my conjecture [17, Section 1]: see Appendix B for a short discussion about how this goes.

(c) A much stronger qualitative theorem is conjecturally true. The following is a restatement of the Local Realization Conjecture of [13].

Conjecture 1.4 Let $M$ be an $\mathcal{A}$-module concentrated in a finite number of degrees. Then for $k \gg 0$, there is no space or spectrum $X$ with

$$
H^{*}(X ; \mathbb{Z} / 2) \simeq M \otimes \Phi(k, k+1) .
$$


In pictures, $M \otimes \Phi(k, k+1)$ looks like

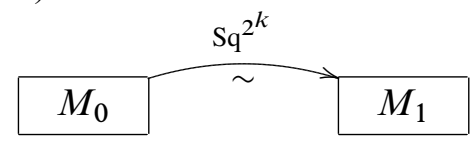

where $M_{i}$ is the $2^{k+i}$-th suspension of $M$.

(d) Statement (a) of the theorem admits a simple straightforward proof that avoids all spectral sequences. Our proofs of both parts will make clear that many other modules are ruled out for topological realization besides those explicitly appearing in the theorem. (The same comment could be made about Schwartz's paper.) There is also a hint, in our discussion of realizing $\Sigma^{2} \Phi(1,3)$ in Section 6 , that more systematic use of Nishida relations might rule out new classes of modules.

(e) Schwartz' theorem holds for all primes. Thus far, we have only worked out the details with mod 2 coefficients, but our work here can certainly be modified for odd primes. It similarly seems likely that our methods here will lead to streamlined proofs of the various other related nonrealization theorems that Schwartz and his students have proved $[18 ; 7 ; 9]$. By using the single Goodwillie tower spectral sequence in our argument here, we have been able to make more delicate use of the unstable module structure of $M$ than does Schwartz, and the author expects that subtle questions about how the Nilpotent and Krull filtrations of $\mathcal{U}$ are reflected as one passes from $H^{*}(X ; \mathbb{Z} / p)$ to $H^{*}\left(\Omega^{n} X ; \mathbb{Z} / p\right)$ can be best approached using our techniques.

Notation 1.5 We use the following notation. $H^{*}(X)$ will mean $H^{*}(X ; \mathbb{Z} / 2)$. If $x \in M$ is an element of a graded vector space, then $\sigma x$ is the corresponding element of the suspended vector space $\Sigma M$. If $X$ is a space, we will write $\Sigma^{-n} X$ for the desuspended suspension spectrum $\Sigma^{-n} \Sigma^{\infty} X$. As in [15], $\mathcal{C}(n, j)$ denotes the space of $j$ little $n$-cubes in a big $n$-cube. This has a free action by the $j$-th symmetric group $\Sigma_{j}$, and, for $X$ a space or spectrum, we let $D_{n, j} X=\left(\mathcal{C}(n, j)_{+} \wedge X^{\wedge j}\right)_{h \Sigma_{j}}$. Note that $D_{1, j} X \simeq X^{\wedge j}$ and $D_{\infty, j} X \simeq X_{h \Sigma_{j}}^{\wedge j}$. By convention, $D_{n, 0} X=S^{0}$ for all $n$ and $X$.

The rest of the paper is organized as follows. For much of it - Sections 2, 3 and 4, supported by Appendix A - we describe some of the general properties of the spectral sequence for computing $H^{*}\left(\Omega^{n} X\right)$. Assuming this material, the proof of Theorem 1.1 is satisfyingly short, and given in Section 5. Illustrating the methods of our proof, in Section 6 we look more carefully at how things go when $\widetilde{H}^{*}(X) \simeq \Sigma^{2} \Phi(1,3)$.

A version of our argument here has been known by the author for nearly a decade; indeed, I gave a talk "A simple proof of Schwartz' nonrealization theorem" at the Midwest Topology conference of October, 23, 1999. I apologize for the delay in 
writing this up, and plead that this project led me to become infatuated with Goodwillie towers. (To be honest, the needed geometric details underpinning the spectral sequence used here were only worked out later in joint work with S Ahearn [2].) I am happy to be reunited with an earlier love: the category $\mathcal{U}$.

The author would like to thank Mark Mahowald, Brayton Gray, and particularly Elmer Rees for aid with Example 1.2. Brayton and Elmer responded to a query of mine posted on the Algebraic topology discussion list [6]: see the messages of June 5, June 6, and August 3, 2008. So Don Davis also deserves thanks for providing this forum.

This research was partially supported by grants from the National Science Foundation.

\section{The Arone-Goodwillie tower of $\Sigma^{\infty} \Omega^{n} X$}

For $n<\infty$, one has a functor sending a based space $X$ to the suspension spectrum $\Sigma^{\infty} \Omega^{n} X$. For $n=\infty$, one similarly has a functor sending a spectrum $X$ to the spectrum $\Sigma^{\infty} \Omega^{\infty} X$. In either case, T Goodwillie's general theory of the calculus of functors $[10 ; 11 ; 12]$ yields natural towers of fibrations

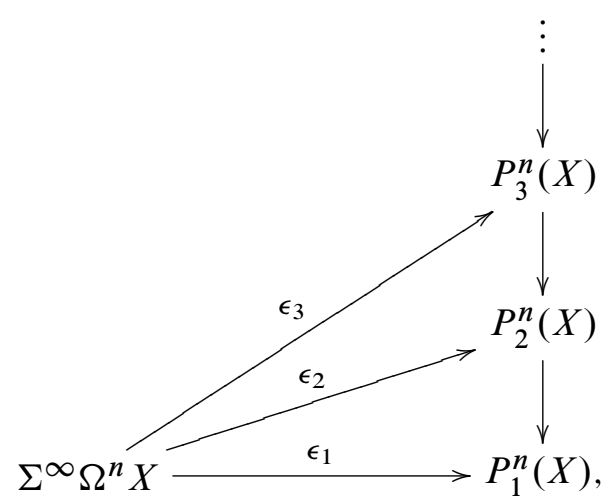

such that the connectivity of the maps $\epsilon_{j}$ increases linearly with $j$ as long as $X$ is $n$-connected if $n<\infty$, and is 0 -connected if $n=\infty$.

Using G Arone's explicit model for this tower [3], properties of these towers were explored in [2].

For $n<\infty$, the spectrum $P_{1}^{n}(X)$ identifies with the spectrum $\Sigma^{-n} X$, so that $\epsilon_{1}$ corresponds to the evaluation map

$$
\Sigma^{\infty} \Omega^{n} X \rightarrow \Sigma^{-n} X
$$


and the fiber of the map $P_{j}^{n}(X) \rightarrow P_{j-1}^{n}(X)$ is naturally equivalent to the spectrum $D_{n, j} \Sigma^{-n} X$. Similarly, when $n=\infty$, the $j$-th fiber is equivalent to $D_{\infty, j} X$, and $\epsilon_{1}$ corresponds to the evaluation map

$$
\Sigma^{\infty} \Omega^{\infty} X \rightarrow X
$$

Applying $H^{*}$ to the towers yields 2nd quadrant spectral sequences. From what we have said above, one can immediately conclude the following.

When $n<\infty$, the spectral sequence converges strongly to $H^{*}\left(\Omega^{n} X\right)$ if $X$ is an $n$-connected space, and has

$$
E_{1}^{-j, *}=\Sigma^{j} H^{*}\left(D_{n, j} \Sigma^{-n} X\right) .
$$

When $n=\infty$, the spectral sequence converges strongly to $H^{*}\left(\Omega^{\infty} X\right)$ if $X$ is a $0-$ connected spectrum, and has

$$
E_{1}^{-j, *}=\Sigma^{j} H^{*}\left(D_{\infty, j} X\right)
$$

For all $n, E_{\infty}^{*, *}$ is the graded object associated to the filtration of $H^{*}\left(\Omega^{n} X\right)$,

$$
\cdots \supseteq F^{-3} H^{*}\left(\Omega^{n} X\right) \supseteq F^{-2} H^{*}\left(\Omega^{n} X\right) \supseteq F^{-1} H^{*}\left(\Omega^{n} X\right),
$$

where $F^{-j} H^{*}\left(\Omega^{n} X\right)=\operatorname{Im}\left\{\epsilon_{j}^{*}: H^{*}\left(P_{j}^{n}(X)\right) \rightarrow H^{*}\left(\Omega^{n} X\right)\right\}$.

The spectral sequences are compatible as $n$-varies. More precisely, the natural evaluation maps

$$
\Sigma^{r} \Sigma^{\infty} \Omega^{n+r} X \rightarrow \Sigma^{\infty} \Omega^{n} X
$$

as well as the natural equivalences (with $X_{n}=\Omega^{\infty} \Sigma^{n} X$ )

$$
\Sigma^{\infty} \Omega^{n} X_{n} \stackrel{\sim}{\rightarrow} \Sigma^{\infty} \Omega^{\infty} X
$$

induce maps of towers, and then spectral sequences.

Remark 2.1 When $n=1$, one recovers the classical Eilenberg-Moore spectral sequence with $E_{1}^{-j, *}=H^{*}(X)^{\otimes j}$. For general $n<\infty$, general Goodwillie calculus considerations imply that the spectral sequence constructed here must necessarily agree with the dual of the spectral sequence studied by V Smirnov in [19, Chapter 6]. 


\section{The mod 2 cohomology of $D_{n, *} X$}

To use our spectral sequence, we need to have a useful description of the bigraded object $H^{*}\left(D_{n, *} X\right)$. In this section, we give this, by constructing various natural operations. It is more traditional to describe $H_{*}\left(D_{n, *} X\right)$ using Dyer-Lashof operations, Browder operations, and the Pontryagin product $[5 ; 4]$, and our operations are easily verified to be appropriately "dual" to these: see Proposition A.1. Because of this, we will be brief with some verifications of properties.

To avoid problems with duals of infinite dimensional vector spaces, in the discussion that follows we make the blanket assumption: for all spectra $X, H_{*}(X)$ is bounded below and is of finite type. This is not a serious restriction, as any spectrum $X$ can be written as hocolim $k X(k)$ where $X(k)$ satisfies this assumption, and then $H^{*}\left(D_{n, *} X\right)=\lim _{k} H^{*}\left(D_{n, *} X(k)\right)$, as profinite bigraded vector spaces.

\subsection{Structure maps}

Definitions 3.1 (a) Let $\epsilon: \Sigma^{r} D_{n+r, j} X \rightarrow D_{n, j} \Sigma^{r} X$ denote the map induced by the evaluation map (2-1). (See Ahearn and Kuhn [2] for an explicit formula.)

(b) Let $\mu: D_{n, i} X \wedge D_{n, j} X \rightarrow D_{n, i+j} X$ denote the map induced by the inclusion $\Sigma_{i} \times \Sigma_{j} \subset \Sigma_{i+j}$.

(c) Let $t: D_{n, i+j} X \rightarrow D_{n, i} X \wedge D_{n, j} X$ denote the composite of the maps

$$
\left(\mathcal{C}(n, i+j)_{+} \wedge X^{\wedge i+j}\right)_{h \Sigma_{i+j}} \rightarrow\left(\mathcal{C}(n, i+j)_{+} \wedge X^{\wedge i+j}\right)_{h \Sigma_{i} \times \Sigma_{j}}
$$

and $\left(\mathcal{C}(n, i+j)_{+} \wedge X^{\wedge i+j}\right)_{h \Sigma_{i} \times \Sigma_{j}} \rightarrow\left(\mathcal{C}(n, i)_{+} \wedge \mathcal{C}(n, j)_{+} \wedge X^{\wedge i+j}\right)_{h \Sigma_{i} \times \Sigma_{j}}$,

where the first map is the transfer associated to $\Sigma_{i} \times \Sigma_{j} \subset \Sigma_{i+j}$ and the second map is induced by the $\Sigma_{i} \times \Sigma_{j}$-equivariant inclusion of spaces

$$
\mathcal{C}(n, i+j) \subset \mathcal{C}(n, i) \times \mathcal{C}(n, j) .
$$

(d) Let $w: D_{n, 2 j} X \rightarrow D_{\infty, 2} D_{n, j} X$ denote the composite of the maps

and

$$
\left(\mathcal{C}(n, 2 j)_{+} \wedge X^{2 j}\right)_{h \Sigma_{2 j}} \rightarrow\left(\mathcal{C}(n, 2 j)_{+} \wedge X^{2 j}\right)_{h \Sigma_{2} i \Sigma_{j}}
$$

$$
\left(\mathcal{C}(n, 2 j)_{+} \wedge X^{2 j}\right)_{h \Sigma_{2}\left\langle\Sigma_{j}\right.} \rightarrow\left(\mathcal{C}(n, j)_{+}^{2} \wedge X^{2 j}\right)_{h \Sigma_{2} \ell \Sigma_{j}},
$$

where the first map is the transfer associated to the inclusion $\Sigma_{2} \succ \Sigma_{j} \subset \Sigma_{2 j}$ and the second map is induced by the $\Sigma_{2}<\Sigma_{j}$-equivariant inclusion of spaces

$$
\mathcal{C}(n, 2 j) \subset \mathcal{C}(n, j)^{2} \text {. }
$$




\subsection{Operations}

Definition 3.2 For $r \geq 0$, define natural operations

$$
\widehat{Q}_{r}: H^{d}\left(D_{n, j} X\right) \rightarrow H^{2 d+r}\left(D_{n, 2 j} X\right)
$$

as follows.

Given $x \in H^{d}(X)$, viewed as a map $x: X \rightarrow \Sigma^{d} H \mathbb{Z} / 2$, we let $\widehat{Q}_{0}(x) \in H^{2 d}\left(D_{\infty, 2} X\right)$ be the composite

$$
D_{\infty, 2} X \stackrel{D_{\infty, 2} x}{\longrightarrow} D_{\infty, 2} \Sigma^{d} H \mathbb{Z} / 2 \stackrel{u}{\rightarrow} \Sigma^{2 d} H \mathbb{Z} / 2,
$$

where $u$ represents the bottom class in $H^{*}\left(D_{\infty, 2} \Sigma^{d} H \mathbb{Z} / 2\right)$.

Given $x \in H^{d}\left(D_{n, j} X\right)$, we let $\widehat{Q}_{0}(x) \in H^{2 d}\left(D_{n, 2 j} X\right)$ be the composite

$$
D_{n, 2 j} X \stackrel{w}{\rightarrow} D_{\infty, 2} D_{n, j} X \stackrel{\hat{Q}_{0}(x)}{\longrightarrow} \Sigma^{2 d} H \mathbb{Z} / 2,
$$

and then, for $r>0$, we let $\widehat{Q}_{r}(x) \in H^{2 d+r}\left(D_{n, 2 j} X\right)$ be the composite

$$
D_{n, 2 j} X \stackrel{\epsilon}{\rightarrow} \Sigma^{-r} D_{n-r, 2 j} \Sigma^{r} X \stackrel{\Sigma^{-r} \widehat{Q}_{0}\left(\Sigma^{r} x\right)}{\longrightarrow} \Sigma^{2 d+r} H \mathbb{Z} / 2
$$

Definition 3.3 Define a natural product

$$
*: H^{*}\left(D_{n, i} X\right) \otimes H^{*}\left(D_{n, j} X\right) \rightarrow H^{*}\left(D_{n, i+j} X\right)
$$

to be the map on cohomology induced by the "transfer" maps

$$
t: D_{n, i+j} X \rightarrow D_{n, i} X \wedge D_{n, j} X \text {. }
$$

Note that, when $n=1$, the $*$-product is the standard shuffle product on the tensor algebra $T H^{*}(X)$.

Definition 3.4 Define a natural coproduct

$$
\Psi: H^{*}\left(D_{n, i+j} X\right) \rightarrow H^{*}\left(D_{n, i} X\right) \otimes H^{*}\left(D_{n, j} X\right)
$$

to be the map on cohomology induced by the maps

$$
\mu: D_{n, i} X \wedge D_{n, j} X \rightarrow D_{n, i+j} X .
$$


Definition 3.5 For $n<\infty$ and $d_{1}+\cdots+d_{j}=d$, define

$$
L_{n-1}: H^{d_{1}}(X) \otimes \cdots \otimes H^{d_{j}}(X) \rightarrow H^{d+(j-1)(n-1)}\left(D_{n, j} X\right)
$$

to be the map on cohomology induced by the map

$$
\epsilon: D_{n, j} X \rightarrow \Sigma^{1-n} D_{1, j} \Sigma^{n-1} X=\Sigma^{(j-1)(n-1)} X^{\wedge j} .
$$

Note that $L_{0}$ is just the usual product in the tensor algebra $T H^{*}(X)$.

The following will be made precise in Appendix A. See Proposition A.1.

Proposition 3.6 In a suitable sense, the cohomology operations $\widehat{Q}_{r}$, *, and $L_{n-1}$ are dual to the homology operations $Q_{r}, *$, and $\lambda_{n-1}$.

\subsection{Some properties of the operations}

Proposition 3.7 The $*$-product and $\Psi$-coproduct makes $H^{*}\left(D_{n, *} X\right)$ into a bigraded bicommutative Hopf algebra.

Proposition 3.8 For all $x \in H^{*}\left(D_{n, j} X\right), \widehat{Q}_{r}(x)=0$ for $r \geq n$.

Proposition 3.9 Under $\epsilon^{*}: H^{*}\left(D_{n, *} \Sigma X\right) \rightarrow H^{*-1}\left(D_{n+1, *} X\right)$, the operations behave as follows.

(i) $\epsilon^{*}$ sends $*$-decomposables to $0: \epsilon^{*}(x * y)=0$ for all $x \in H^{*}\left(D_{n, i} \Sigma X\right)$ and $y \in H^{*}\left(D_{n, j} \Sigma X\right)$, with $i \geq 1$ and $j \geq 1$. Similarly, the image of $\epsilon^{*}$ is contained in the $\Psi$-primitives.

(ii) $\epsilon^{*}$ commutes with the $\widehat{Q}$ operations: $\epsilon^{*}\left(\widehat{Q}_{r}(\sigma x)\right)=\widehat{Q}_{r+1}(x)$.

(iii) $\epsilon^{*}$ commutes with the $L$ operations: for all $x_{1}, \ldots, x_{k} \in H^{*}(X)$,

$$
\epsilon^{*}\left(L_{n-1}\left(\sigma x_{1} \otimes \cdots \otimes \sigma x_{k}\right)\right)=L_{n}\left(x_{1} \otimes \cdots \otimes x_{k}\right) .
$$

Proof Parts (ii) and (iii) are clear from the definition, and part (i) is only slightly less so. For more detail about (i), see Example 6.7 of [2].

Proposition 3.10 For all $x \in H^{*}(X)^{\otimes k}, \widehat{Q}_{n-1}\left(L_{n-1}(x)\right)=L_{n-1}(x \otimes x)$.

Proof By parts (ii) and (iii) of the last proposition, this reduces to the case when $n=1$, where it reads $\hat{Q}_{0}(x)=x \otimes x$, for $x \in H^{*}\left(X^{\wedge k}\right)$, and this is clear from the definition of $\hat{Q}_{0}$. 
Proposition 3.11 For all $x, y \in H^{d}\left(D_{n, j} X\right)$, the following identities hold.

(i) $\widehat{Q}_{0}(x+y)=\widehat{Q}_{0}(x)+\widehat{Q}_{0}(y)+x * y$.

(ii) $\widehat{Q}_{r}(x+y)=\widehat{Q}_{r}(x)+\widehat{Q}_{r}(y)$, for all $r>0$.

(iii) $x * x=0$.

See Appendix A for a proof.

Proposition 3.12 For all $n \geq 2$, the kernel of $L_{n-1}$ : $T H^{*}(X) \rightarrow H^{*}\left(D_{n, *} X\right)$ is the span of the shuffle product decomposables.

Proof This is dual to the well-known statement that the image of $\epsilon_{*}: H_{*}\left(D_{n, *} X\right) \rightarrow$ $T H_{*}(X)$ is the vector space of primitives, which identifies as the free restricted Lie algebra generated by $H_{*}(X)$. Note that Proposition 3.9(i) implies that the kernel is at least as big as claimed.

One has Adem relations among the $\widehat{Q}_{r}$.

\section{Proposition 3.13}

$$
\hat{Q}_{r} \hat{Q}_{s}(x)=\sum_{j}\left(\begin{array}{c}
j-r \\
2 j-r-s
\end{array}\right) \hat{Q}_{r+2 s-2 j} \hat{Q}_{j}(x) .
$$

This follows from the homology Adem relations, using Proposition A.1. Similarly, the calculation of $H_{*}\left(D_{n, *} X\right)$ as in [5;4] implies the next theorem.

Theorem 3.14 Using the *-product, $H^{*}\left(D_{n, *} X\right)$ is the graded commutative algebra generated by the elements of the form $\widehat{Q}_{r_{1}} \ldots \widehat{Q}_{r_{l}} L_{n-1}\left(x_{1} \otimes \cdots \otimes x_{k}\right)$, subject to the relations listed in Propositions 3.8, 3.11, 3.12 and 3.13.

Finally, we have Nishida relations. Compare with [16, page 40], [4, Theorem II.3.5] and [14, Proposition 6.12].

Proposition 3.15 For all $x \in H^{d}\left(D_{n, j} X\right)$, the following identities hold.

(i) $\mathrm{Sq}^{s} \hat{Q}_{0}(x)=\sum_{t}\left(\begin{array}{c}d-t \\ s-2 t\end{array}\right) \hat{Q}_{s-2 t}\left(\mathrm{Sq}^{t} x\right)+\sum_{t<s / 2} \mathrm{Sq}^{t} x * \mathrm{Sq}^{s-t} x$.

(ii) $\mathrm{Sq}^{s} \widehat{Q}_{r}(x)=\sum_{t}\left(\begin{array}{c}d+r-t \\ s-2 t\end{array}\right) \hat{Q}_{r+s-2 t}\left(\mathrm{Sq}^{t} x\right)$.

See Appendix A for more about this. 


\section{Some properties of the spectral sequence for $H^{*}\left(\Omega^{n} X\right)$}

Here we collect some basic properties of the spectral sequences arising from the towers of Section 2. From [2], we learn the following.

Proposition 4.1 The spectral sequence is a spectral sequence of differential graded Hopf algebras, with the product and coproduct structure on $E_{1}$ given by the $*$ and $\Psi$, converging to the usual Hopf algebra structure on $H^{*}\left(\Omega^{n} X\right)$.

From the geometric construction of the spectral sequence, we deduce the next proposition.

Proposition 4.2 The spectral sequence is a spectral sequence of $\mathcal{A}$-modules, with $\mathcal{A}$ acting columnwise on $E_{1}$ in the evident way, and converging to the usual $\mathcal{A}-$ module structure on $H^{*}\left(\Omega^{n} X\right)$. In particular, $F^{-j} H^{*}\left(\Omega^{n} X\right)$ is a sub-A-module of $H^{*}\left(\Omega^{n} X\right)$ for all $j$.

Finally we determine the differential $d_{1}$ from the -2 line to the -1 line. In other words, we determine the homomorphism

$$
d_{1}: \Sigma^{2} H^{*}\left(D_{n, 2} \Sigma^{-n} X\right) \rightarrow \Sigma H^{*}\left(\Sigma^{-n} X\right),
$$

induced by the connecting map $\delta$ in the cofibration sequence

$$
D_{n, 2} \Sigma^{-n} X \rightarrow P_{2}^{n}(X) \rightarrow \Sigma^{-n} X \stackrel{\delta}{\rightarrow} \Sigma D_{n, 2} \Sigma^{-n} X .
$$

Proposition 4.3 For $x, y \in H^{*}(X)$ we have the following formulae.

(i) $d_{1}\left(\sigma^{2} L_{n-1}\left(\sigma^{-n} x \otimes \sigma^{-n} y\right)\right)=\sigma^{1-n}(x \cup y)$.

(ii) $d_{1}\left(\sigma^{2} \widehat{Q}_{r}\left(\sigma^{-n} x\right)\right)=\sigma^{1-n} \mathrm{Sq}^{r+|x|-n+1}(x)$.

(iii) $d_{1}(x * y)=0$.

Proof Formula (iii) is clear, as $d_{1}$ is a derivation.

Formula (i) reduces to the case when $n=1$, where it becomes the well-known formula

$$
d_{1}(x \otimes y)=x \cup y
$$

in the bar construction associated to the Eilenberg-Moore spectral sequence.

Formula (ii) reduces to the case when $r=0$, and then to the case when $n=\infty$, where we wish to show that, for $X$ a 0 -connected spectrum and $x \in H^{d}(X)$,

$$
d_{1}\left(\sigma^{2} \hat{Q}_{0}(x)\right)=\sigma \mathrm{Sq}^{d+1} x \text {. }
$$


As the left side of the equation is natural, there must be an element $a \in \mathcal{A}^{d+1}$ such that

$$
d_{1}\left(\sigma^{2} \hat{Q}_{0}(x)\right)=\sigma a x .
$$

To show that $a$ must be $\mathrm{Sq}^{d+1}$, we consider the universal case: the spectral sequence when $X=\Sigma^{d} H \mathbb{Z} / 2$. In this case, $\Sigma^{\infty} \Omega^{\infty} X=K(\mathbb{Z} / 2, d)$, and so the spectral sequence converges to $H^{*}(K(\mathbb{Z} / 2, d))$, and $\Sigma^{-1} E_{\infty}^{-1, *}$ will be the image of $\epsilon^{*}: H^{*}\left(\Sigma^{d} H \mathbb{Z} / 2\right) H^{*}(K(\mathbb{Z} / 2, d))$. This image is just the cyclic $\mathcal{A}$-module generated by the fundamental class $u_{d} \in H^{d}(K(\mathbb{Z} / 2, d))$. Since $\operatorname{Sq}^{d+1} u_{d}=0 \in$ $H^{*}(K(\mathbb{Z} / 2, d)), \sigma \mathrm{Sq}^{d+1} u \in E_{1}^{-1, *}$ must be a boundary, where $u$ is the bottom class of $\Sigma^{d} \mathcal{A}$. For connectivity reasons, the only way for this to happen is if $d_{1}\left(\sigma^{2} \hat{Q}_{0}(u)\right)=$ $\sigma \mathrm{Sq}^{d+1} u$.

Remark 4.4 The proposition should be compared to the homology formulae in [19, 6.2], in particular, Smirnov's formula on page 124, three lines before his second theorem.

Corollary 4.5 In the spectral sequence computing $H^{*}\left(\Omega^{n} X\right)$ with $X$ an $n$-connected space, $\Sigma^{-1} E_{2}^{-1, *}$ will be the maximal unstable quotient of

$$
\Sigma^{-n}\left(H^{*}(X) /(\cup \text {-decomposables })\right) .
$$

Even more is true if $\widetilde{H}^{*}(X) \simeq \Sigma^{n} M$ with $M \in \mathcal{U}$, and has no nontrivial cup products: then $E_{3}^{-1, *}=E_{2}^{-1, *}=E_{1}^{-1, *}$, and $E_{2}^{-2, *}=E_{1}^{-2, *}$.

Proof The first statement follows evidently from the previous proposition. In the situation of the second statement, the assumption then tells us that $d_{1}: E_{1}^{-2, *} \rightarrow E_{1}^{-1, *}$ is identically zero. Since $E_{1}^{-3, *}$ is spanned by $*$-decomposables, the fact that $d_{1}$ is a derivation allows us to conclude that $d_{1}: E_{1}^{-3, *} \rightarrow E_{1}^{-2, *}$ is also identically zero. Thus we have that both $E_{2}^{-2, *}=E_{1}^{-2, *}$ and $E_{2}^{-1, *}=E_{1}^{-1, *}$. It follows that $E_{2}^{-3, *}$ is again spanned by algebra decomposables, and so, as before, we conclude that $d_{2}: E_{2}^{-3, *} \rightarrow E_{2}^{-1, *}$ is identically zero.

There is a similar corollary in the $n=\infty$ case.

Corollary 4.6 In the spectral sequence computing $H^{*}\left(\Omega^{\infty} X\right)$ with $X$ a 0 -connected spectrum, $\Sigma^{-1} E_{2}^{-1, *}$ will be the unstable quotient of $H^{*}(X)$. Even more is true if $\widetilde{H}^{*}(X) \simeq M$ with $M \in \mathcal{U}$ : then $E_{3}^{-1, *}=E_{2}^{-1, *}=E_{1}^{-1, *}$, and $E_{2}^{-2, *}=E_{1}^{-2, *}$. 


\section{Proof of Theorem 1.1}

Recall the assumptions on $M$ in the theorem. We have numbers $l \leq m$ such that $M^{s} \neq 0$ only if $l \leq s \leq m$. The statement that $M$ is not the desuspension of an unstable module means precisely that there exists $x \in M$ such that $\mathrm{Sq}^{|x|} x \neq 0$. We fix such an element and let $d=|x|$, so that $l \leq d \leq 2 d \leq m$.

Assuming that $2^{k}>m-l$, it is easily verified that $M \otimes \Phi(k, k+2)$ is the module as pictured in $(1-1)$ :

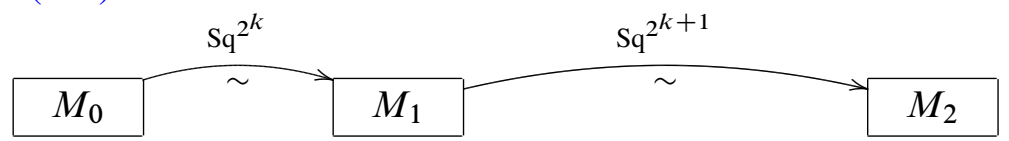

where $M_{i}=M \otimes\left\langle t^{2^{k+i}}\right\rangle$. We let $a=x \otimes t^{2^{k}} \in M_{0}, b=x \otimes t^{2^{k+1}} \in M_{1}$, and $c=x \otimes t^{2^{k+2}} \in M_{2}$. Thus $|a|=d+2^{k},|b|=d+2^{k+1},|c|=d+2^{k+2}, \mathrm{Sq}^{2^{k}} a=b$, $\mathrm{Sq}^{2^{k+1}} b=c$, and $\mathrm{Sq}^{d} c \neq 0$.

With this notation, we give the quick proof of Theorem 1.1(a). If $2^{k}>m-l$, we show that there is no unstable algebra structure on $M \otimes \Phi(k, k+2)$, so that there can be no space $X$ such that $\tilde{H}^{*}(X) \simeq M \otimes \Phi(k, k+2)$.

The proof of this is simple. We begin with the calculation:

$$
\begin{aligned}
\mathrm{Sq}^{2^{k}}(a \cup b) & =b \cup b & & \text { (by the Cartan formula) } \\
& =\mathrm{Sq}^{d+2^{k+1}} b & & \text { (by the Restriction axiom) } \\
& =\mathrm{Sq}^{d} c \neq 0 . & &
\end{aligned}
$$

Here the third equality follows from the calculation in $M \otimes \Phi(k, k+2)$ that

$$
\mathrm{Sq}^{d+2^{k+1}}\left(x \otimes t^{2^{k+1}}\right)=\mathrm{Sq}^{d} x \otimes t^{2^{k+2}}=\mathrm{Sq}^{d}\left(x \otimes t^{2^{k+2}}\right) \text {. }
$$

Thus $a \cup b \neq 0$. But $|a \cup b|=2 d+3 \cdot 2^{k}$, which we claim is a degree in the "gap" between $M_{1}$ and $M_{2}$, so that $a \cup b=0$, giving us a contradiction. In other words, we claim that

$$
m+2^{k+1}<2 d+3 \cdot 2^{k}<l+2^{k+2} .
$$

The first inequality follows by adding $2^{k+1}$ to the inequalities

$$
m<l+2^{k} \leq 2 d+2^{k},
$$

while the second inequality follows by adding $3 \cdot 2^{k}$ to the inequalities

$$
2 d \leq m<l+2^{k} \text {. }
$$

We now begin the longer proof of Theorem 1.1(b). So let $n>0$, and suppose that $\widetilde{H}^{*}(X) \simeq \Sigma^{n} M \otimes \Phi(k, k+2)$, and has trivial cup products. We can assume that 
$X$ is a CW complex. For technical reasons, rather than working with $X$, we work with the quotient $Y=X / X_{d+n+2^{k}-1}$. This will be needed to ensure that $\sigma \mathrm{Sq}^{d}(c) \in$ $E_{1}^{-1,2 d+2^{k}+1}$ is not in the image of $d_{3}$ : see Lemma 5.3. (Replacing $X$ by $Y$ would not be needed if $d \leq 2 l$.)

Since $H^{*}(Y) \rightarrow H^{*}(X)$ is an isomorphism for $*>d+n+2^{k}$ and is epic if $*=d+n+2^{k}$, one easily deduces that $\widetilde{H}^{*}(Y) \simeq \Sigma^{n} N$ with $N \in \mathcal{U}$, still has trivial cup products, and $N$ is as pictured:

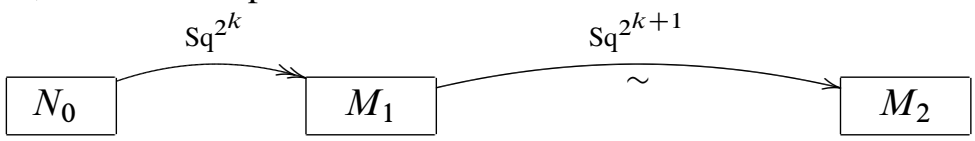

where $N_{0}, M_{1}$, and $M_{2}$ are nonzero only in degrees in the intervals $\left[d+2^{k}, m+2^{k}\right]$, $\left[l+2^{k+1}, m+2^{k+1}\right]$, and $\left[l+2^{k+2}, m+2^{k+2}\right]$.

Choosing a "new" $a \in N_{0}$ mapping onto the "old" $a \in M_{0}$, we have as before: $|a|=d+2^{k},|b|=d+2^{k+1},|c|=d+2^{k+2}, \mathrm{Sq}^{2^{k}} a=b, \mathrm{Sq}^{2^{k+1}} b=c$, and $\mathrm{Sq}^{d} c \neq 0$.

We assume the inequality

$$
2^{k}>4 m-2 l+2 n-2,
$$

and we show that this leads to a contradiction by showing that $H^{*}\left(\Omega^{n} Y\right)$, as computed by our spectral sequence, can not admit an unstable algebra structure.

As a first observation, we note that Corollary 4.5 applies, so that $E_{3}^{-1, *}=E_{2}^{-1, *}=$ $E_{1}^{-1, *}$, and $E_{2}^{-2, *}=E_{1}^{-2, *}$.

A picture of $\Sigma^{-1} E_{1}^{-1, *}$ is given by (5-2), and is all permanent cycles. Thus there exist $\alpha \in H^{d+2^{k}}\left(\Omega^{n} Y\right), \beta \in H^{d+2^{k+1}}\left(\Omega^{n} Y\right)$, and $\gamma \in H^{d+2^{k+2}}\left(\Omega^{n} Y\right)$, respectively represented by $a, b$, and $c$, and we have $\mathrm{Sq}^{2^{k}} \alpha=\beta$, and $\mathrm{Sq}^{2^{k+1}} \beta=\gamma$.

A picture of the additive structure of $\Sigma^{-2} E_{1}^{-2, *}$ in degrees less than $2 l+2^{k+2}$ is given by

$$
N_{0} \cdot N_{0} \quad N_{0} \cdot M_{1}
$$

where $N_{0} \cdot N_{0}$ denotes the span of all elements of the form $\widehat{Q}_{r}(x), x * y$, or $L_{n-1}(x \otimes y)$ with $x, y \in N_{0}$, and $N_{0} \cdot M_{1}$ denotes the span of $x * y$ and $L_{n-1}(x \otimes y)$ with $x \in N_{0}$ and $y \in M_{1}$.

$N_{0} \cdot N_{0}$ is nonzero only in the interval $\left[2 d+2^{k+1}, 2 m+2^{k+1}+n-1\right]$, and includes the element $\hat{Q}_{0}(a)$ in lowest degree. As $E_{2}^{-2, *}=E_{1}^{-2, *}$, this is a permanent cycle, and so represents an element $\delta \in H^{2 d+2^{k+1}}\left(\Omega^{n} Y\right)$. 
$N_{0} \cdot M_{1}$ is nonzero only in the interval $\left[l+d+3 \cdot 2^{k}, 2 m+3 \cdot 2^{k}+n-1\right]$, and includes the element $a * b$, which represents $\alpha \cup \beta \in H^{2 d+3 \cdot 2^{k}}\left(\Omega^{n} Y\right)$.

Lemma $5.1 \mathrm{Sq}^{2^{k}} \delta=\alpha \cup \beta$.

Proof Our constraint (5-3) implies that $2^{k}-2 d \geq n$ and also that $2^{k-1}>m$. Using these inequalities, one easily checks that the formula for $\operatorname{Sq}^{2^{k}} \widehat{Q}_{0}(a)$ given by Proposition 3.15 simplifies to yield

$$
\mathrm{Sq}^{2^{k}} \hat{Q}_{0}(a)=a * \mathrm{Sq}^{2^{k}} a=a * b .
$$

As both $\mathrm{Sq}^{2^{k}} \delta$ and $\alpha \cup \beta$ are represented by $a * b$, it follows that $\mathrm{Sq}^{2^{k}} \delta-\alpha \cup \beta$ is represented by something in bidegree $\left(-1,2 d+3 \cdot 2^{k}+1\right)$. But there is nothing nonzero in this bidegree because (5-3) implies that $2^{k}>m-l$, and this then implies that $m+2^{k+1}<2 d+3 \cdot 2^{k}<l+2^{k+2}$.

Lemma 5.2 $\operatorname{Sq}^{2}(\alpha \cup \beta)=\mathrm{Sq}^{d} \gamma$.

Proof Just as in (5-1), we have $\mathrm{Sq}^{2^{k}}(\alpha \cup \beta)=\beta^{2}=\mathrm{Sq}^{d+2^{k+1}} \beta=\mathrm{Sq}^{d} \gamma$.

Lemma 5.3 $\operatorname{Sq}^{d} \gamma \neq 0$.

Proof The lowest degree differential with potentially nonzero image in the -1 -line would be

$$
d_{3}: E_{3}^{-4,4 d+2^{k+2}+4} \rightarrow E_{3}^{-1,4 d+2^{k+2}+2} .
$$

Thus $\mathrm{Sq}^{d} c \in E_{3}^{-1,2 d+2^{k+2}+1}$ is not a boundary.

Corollary $5.4 \mathrm{Sq}^{2^{k}} \mathrm{Sq}^{2^{k}} \delta \neq 0$.

We will now use the next lemma to show that $\mathrm{Sq}^{2^{k}} \mathrm{Sq}^{2^{k}} \delta=0$ if our numerical constraint (5-3) holds, and this contradiction will finish the proof of Theorem 1.1(b). Let $\mathcal{A}(k)$ be the subalgebra of $\mathcal{A}$ generated by $\mathrm{Sq}^{1}, \ldots, \mathrm{Sq}^{2^{k}}$.

Lemma 5.5 [17, Lemma 2.6] $\mathrm{Sq}^{2^{k}} \mathrm{Sq}^{2^{k}} \in \mathcal{A}(k-1) \mathrm{Sq}^{2^{k}} \mathcal{A}(k-1)$.

Corollary 5.6 $\mathrm{Sq}^{2^{k}} \mathrm{Sq}^{2^{k}} \delta=0$. 
Proof This is a calculation taking place in $F^{-2} H^{*}\left(\Omega^{n} Y\right)$, which in the relevant degrees has additive structure that looks like:

$$
M_{1}+N_{0} \cdot N_{0}
$$

$$
N_{0} \cdot M_{1}
$$$$
M_{2}+M_{1} \cdot M_{1}
$$

The element $\delta \in M_{1}+N_{0} \cdot N_{0}$, while $\mathrm{Sq}^{2^{k}} \mathrm{Sq}^{2^{k}} \delta \in M_{2}+M_{1} \cdot M_{1}$. By the lemma, if both of the gaps pictured span greater than $2^{k-1}$ degrees, then the corollary would follow.

The span of the first gap equals

(the bottom degree of $N_{0} \cdot M_{1}$ ) - (the top degree of $M_{1}+N_{0} \cdot N_{0}$ )

$$
=\left(d+l+3 \cdot 2^{k}\right)-\left(2 m+2^{k+1}+n-1\right)=2^{k}+d+l-2 m-n+1 .
$$

The span of the second gap equals

(the bottom degree of $M_{2}+M_{1} \cdot M_{1}$ ) - (the top degree of $N_{0} \cdot M_{1}$ )

$$
=\left(l+2^{k+2}\right)-\left(2 m+3 \cdot 2^{k}+n-1\right)=2^{k}+l-2 m-n+1 .
$$

Thus both gaps have spans bigger than $2^{k-1}$ if

so that

$$
\begin{gathered}
2^{k}+l-2 m-n+1>2^{k-1}, \\
2^{k-1}>2 m-l+n-1,
\end{gathered}
$$

which is our constraint (5-3).

\section{Realizing $\Sigma^{2} \Phi(1,3)$}

Suppose that $\tilde{H}^{*}(X) \simeq \Sigma^{2} \Phi(1,3)$, so there exist nonzero elements $a \in H^{4}(X)$, $b \in H^{6}(X)$, and $c \in H^{10}(X)$ such that $\mathrm{Sq}^{2} a=b$ and $\mathrm{Sq}^{4} b=c$. Using the spectral sequence converging to $H^{*}\left(\Omega^{2} X\right)$ as in the last section, we prove the following.

Proposition 6.1 In this case, $a \cup b=c$ must hold.

Proof Repressing some suspensions from the notation, Figure 1 shows all of $E_{1}^{*, *}$ in total degree less than or equal to 8 , in the spectral sequence converging to $H^{*}\left(\Omega^{2} X\right)$.

The only possible differential here is $d_{1}\left(L_{1}(a \otimes b)\right)=c$ which, by Proposition 4.3, happens exactly when $a \cup b=c \in H^{*}(X)$. Assuming this does not happen, through degree $8, F^{-2} H^{*}\left(\Omega^{2} X\right)$ would have a basis given by elements $1, \alpha, \beta, \delta, \epsilon, \alpha \cup \beta$, $\lambda, \gamma$, and $\omega$, in respective degrees $0,2,4,4,5,6,7,8$, and 8 , and represented by 1 , 


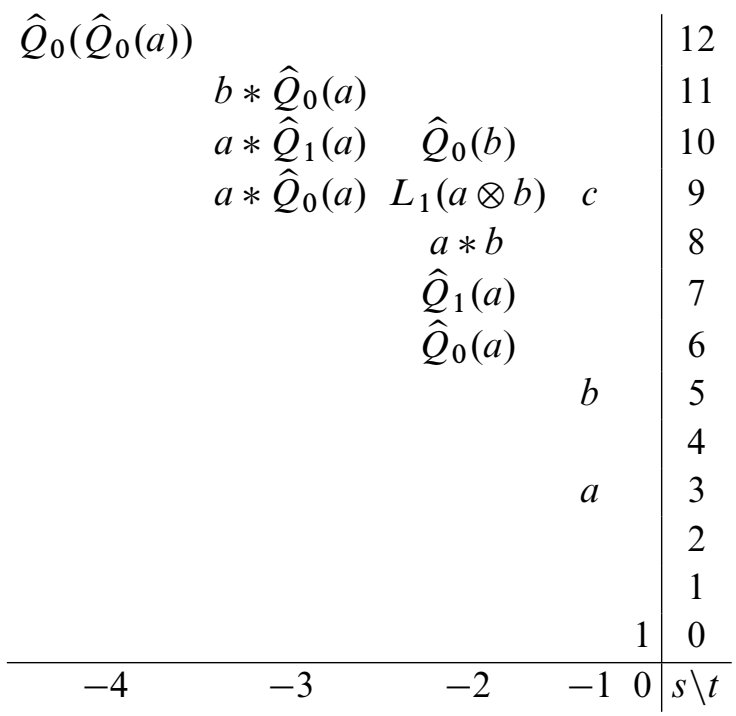

Figure 1: $E_{1}^{s, t}\left(\Omega^{2} X\right)$ when $\widetilde{H}^{*}(X) \simeq \Sigma^{2} \Phi(1,3)$

$a, b, \hat{Q}_{0}(a), \hat{Q}_{1}(a), a * b, L_{1}(a \otimes b), c$, and $\hat{Q}_{0}(b)$. The structure of $\Phi(1,3)$ shows that $\gamma=\beta^{2}=\alpha^{4}$. Furthermore, the arguments in the last section show that $\mathrm{Sq}^{2} \mathrm{Sq}^{2} \delta=\gamma \neq 0$.

The relation $\mathrm{Sq}^{2} \mathrm{Sq}^{2}=\mathrm{Sq}^{1} \mathrm{Sq}^{2} \mathrm{Sq}^{1}$ then implies that $\mathrm{Sq}^{1} \delta \neq 0$. However, the Nishida formula, Proposition 3.15, implies that $\mathrm{Sq}^{1} \hat{Q}_{0}(a)=0$, and thus $\mathrm{Sq}^{1} \delta=0$. This contradiction implies that $d_{1}\left(L_{1}(a \otimes b)\right)=c$ must have been true, so that $a \cup b=c \in H^{10}(X), \lambda \in H^{7}\left(\Omega^{2} X\right)$ doesn't exist, and $\gamma=0 \in H^{8}\left(\Omega^{2} X\right)$.

\section{Appendix A More proofs of the properties of the operations}

Proof of Proposition 3.11 Thanks to Proposition 3.9(i), formula (ii) follows from the formula (i). Letting $x=y$ in (i) implies (iii).

To prove (i), given $x, y \in H^{d}\left(D_{n, j} X\right), \widehat{Q}_{0}(x+y)$ is represented by the composite

$$
D_{n, 2 j} X \stackrel{w}{\rightarrow} D_{\infty, 2} D_{n, j} X \stackrel{D_{\infty, 2}(x+y)}{\longrightarrow} D_{\infty, 2} \Sigma^{d} H \mathbb{Z} / 2 \stackrel{u}{\rightarrow} \Sigma^{2 d} H \mathbb{Z} / 2 .
$$

It is standard [4, Corollary II.1.6] that, given $x, y: Y \rightarrow Z, D_{\infty, 2}(x+y)$ decomposes as the sum of $D_{\infty, 2}(x), D_{\infty, 2}(y)$, and the composite

$$
D_{\infty, 2} Y \stackrel{t}{\rightarrow} Y^{\wedge 2} \stackrel{x \wedge y}{\rightarrow} Z^{\wedge 2} \stackrel{\mu}{\rightarrow} D_{\infty, 2} Z .
$$


It follows that $\widehat{Q}_{0}(x+y)=\widehat{Q}_{0}(x)+\widehat{Q}_{0}(y)$ plus the composite

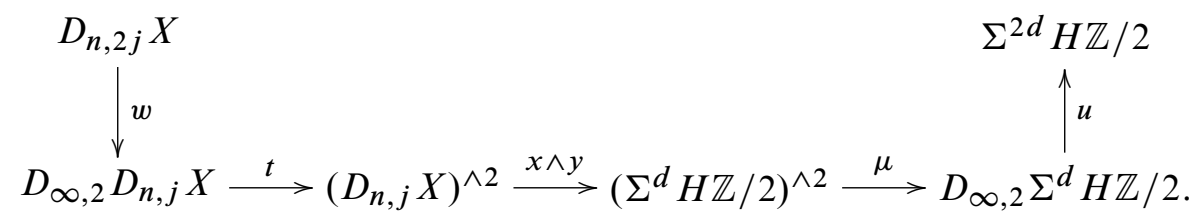

But this last map is just $x * y$, as there is a commutative diagram

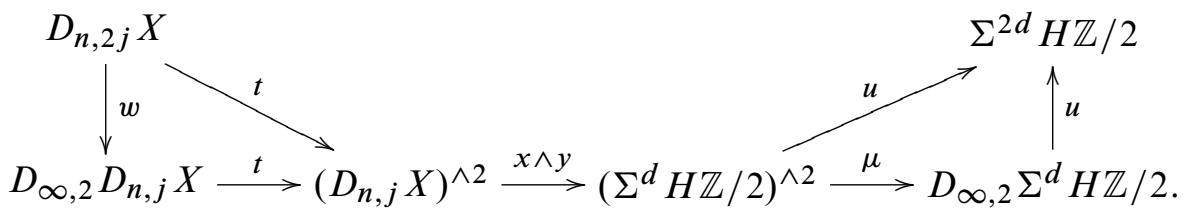

Here the left triangle commutes due to the transitivity of the transfer with respect to the inclusions $\left(\Sigma_{j}\right)^{2} \subset \Sigma_{2} \prec \Sigma_{j} \subset \Sigma_{2 j}$.

We now make precise the "duality" proposition Proposition 3.6. In the following proposition, given $y, z \in H_{*}(X), Q_{r}(y), y * z, \lambda_{n-1}(y, z) \in H_{*}\left(D_{n, 2} X\right)$ denote the usual elements under the Dyer-Lashof operation $Q_{r}$, the Pontryagin product $*$, and the Browder operation $\lambda_{n-1}$ of [5, Part III].

Proposition A.1 Let $\langle x, y\rangle$ denote the cohomology/homology pairing. For $n>1$, given $w, x \in H^{*}(X)$ and $y, z \in H_{*}(X)$, the following formulae hold.

(a) $\left\langle\widehat{Q}_{r} x, Q_{s} y\right\rangle= \begin{cases}\langle x, y\rangle & \text { if } r=s \\ 0 & \text { otherwise. }\end{cases}$

(b) $\left\langle\hat{Q}_{r} x, y * z\right\rangle= \begin{cases}\langle x, y\rangle\langle x, z\rangle & \text { if } r=0 \\ 0 & \text { otherwise. }\end{cases}$

(c) $\left\langle\widehat{Q}_{r} x, \lambda_{n-1}(y, z)\right\rangle=0$.

(d) $\left\langle w * x, Q_{s} y\right\rangle= \begin{cases}\langle w, y\rangle\langle x, y\rangle & \text { if } s=0 \\ 0 & \text { otherwise. }\end{cases}$

(e) $\langle w * x, y * z\rangle=\langle w, y\rangle\langle x, z\rangle+\langle w, z\rangle\langle x, y\rangle$.

(f) $\left\langle w * x, \lambda_{n-1}(y, z)\right\rangle=0$.

(g) $\left\langle L_{n-1}(w \otimes x), Q_{s} y\right\rangle=0$.

(h) $\left\langle L_{n-1}(w \otimes x), y * z\right\rangle=0$.

(i) $\left\langle L_{n-1}(w \otimes x), \lambda_{n-1}(y, z)\right\rangle=\langle w, y\rangle\langle x, z\rangle+\langle w, z\rangle\langle x, y\rangle$. 
Sketch proof The behavior of the homology operations under the evaluation

$$
\epsilon: \Sigma^{s} D_{n+s, 2} X \rightarrow D_{n, 2} \Sigma^{s} X
$$

is well known [5, Theorem III.1.4]: $\epsilon_{*}\left(\sigma^{s} Q_{n+s} y\right)=Q_{s}\left(\sigma^{s} y\right), \epsilon_{*}\left(\sigma^{s} y * z\right)=0$, and $\epsilon_{*}\left(\sigma^{s} \lambda_{n+s-1}(y, z)\right)=\lambda_{n-1}\left(\sigma^{s} y, \sigma^{s} z\right)$. Note in particular, that, under $\epsilon: D_{n, 2} X \rightarrow$ $\Sigma^{n-1} X \wedge X$, one has $\epsilon_{*}\left(\lambda_{n-1}(y, z)\right)=\sigma^{n-1} y \otimes z+z \otimes y$.

Similarly, the behavior under $t: D_{n, 2} X \rightarrow X \wedge X$ is easy to describe: $t_{*}\left(Q_{s} y\right)=0$, $t_{*}(y * z)=y \otimes z+z \otimes y$, and $t_{*}\left(\lambda_{n-1}(y, z)\right)=0$.

Using this information, the various formulae are easily verified, using the naturality of the cohomology/homology pairing.

Proof of Proposition 3.15 Proposition 3.9(i) again implies that the formula when $r>0$ follows from the formula when $r=0$. Furthermore, by the construction of the operations, we can assume that $n=\infty$ and $j=1$, and so one just needs to verify (i) for $\mathrm{Sq}^{s} \hat{Q}_{0}(x) \in H^{2 d+s}\left(D_{\infty, 2} X\right)$.

This can be proved in various ways. One is to use the previous proposition together with the usual Nishida relations.

Another approach goes as follows. One verifies (i) for various sorts of spectra $X$.

If $X$ is a suspension spectrum, then the cohomology of $D_{\infty, 2} X$ is detected by the two maps $X \wedge X \rightarrow D_{\infty, 2} X$ and $B \mathbb{Z} / 2_{+} \wedge X \rightarrow D_{\infty, 2} X$, and one checks that the elements on both sides of formula (i) map to the same elements under these detection maps.

If $X=S^{-c}$, then $D_{\infty, 2} X=\Sigma^{-c} \mathbb{R} P_{-c}^{\infty}$, and one can directly check the formula, working within the $\mathcal{A}$-module $\mathbb{Z} / 2\left[t, t^{-1}\right]$.

If (i) is true for $x \in H^{*}(X)$ and $y \in H^{*}(Y)$, then it is true for $x \otimes y \in H^{*}(X \wedge Y)$. To see this, one uses the map

$$
D_{\infty, 2}(X \wedge Y) \rightarrow D_{\infty, 2} X \wedge D_{\infty, 2} Y
$$

which sends $\widehat{Q}_{0}(x) \otimes \widehat{Q}_{0}(y)$ to $\widehat{Q}_{0}(x \otimes y)$.

If (i) is true for spectra $X_{c}$ and $X=\operatorname{hocolim}_{c} X_{c}$ then (i) is true for $X$. This follows since then $H^{*}\left(D_{\infty, 2} X\right)=\lim _{c} H^{*}\left(D_{\infty, 2} X_{c}\right)$ (using our standing finite type hypothesis).

Assembling all these special cases yields the formula for general spectra $X$, as $X \simeq$ hocolim $_{c} S^{-c} \wedge \Sigma^{\infty} X_{c}$, where $X_{c}$ is the $c$-th space of $X$. 


\section{Appendix B The nonrealization conjecture of [13]}

Following [13; 17], we review how Theorem 1.1 implies:

Theorem B.1 If $H^{*}(Z)$ is a finitely generated $\mathcal{A}$-module, then it is a finite dimensional $\mathbb{Z} / 2-$ vector space.

Sketch proof Let $\bar{T}: \mathcal{U} \rightarrow \mathcal{U}$ be the reduced Lannes functor, left adjoint tensoring with $\widetilde{H}^{*}(B \mathbb{Z} / 2)$. Let $\Delta$ : Spaces $\rightarrow$ Spaces be defined by $\Delta(Z)=\operatorname{Map}(B \mathbb{Z} / 2, Z) / Z$, where $Z$ embeds in $\operatorname{Map}(B \mathbb{Z} / 2, Z)$ as the space of constant maps. Under good circumstances, $\bar{T} H^{*}(Z) \simeq H^{*}(\Delta(Z))$.

Suppose that $H^{*}(Z)=L$ is infinite, but finitely generated over $\mathcal{A}$. Replacing $Z$ and $L$ by their suspensions if needed, we can assume that "good circumstances" will hold. As $L$ is a finitely generated $\mathcal{A}$-module, $\bar{T}^{i} L$ will again be finitely generated for all $i$, and $\bar{T}^{l} L=0$ for some $l$. Since $L$ is also infinite, the smallest such $l$ will be at least 2 . Choosing this smallest $l$, let $Y=\Delta^{l-2}(Z)$. Then $N=H^{*}(Y)=\bar{T}^{l-2} H^{*}(Z)$ will still be infinite and finitely generated over $\mathcal{A}$, but now also $\bar{T}^{2} N=0$. (These reductions are made in [13].)

Now we use a structure theorem: $N \in \mathcal{U}$ is finitely generated over $\mathcal{A}$ and satisfies $\bar{T}^{2} N=0$ if and only if it fits into an exact sequence in $\mathcal{U}$ of the form

$$
0 \rightarrow A \rightarrow N \rightarrow M \otimes \Phi(j, \infty) \rightarrow B \rightarrow 0
$$

for some finite dimensional unstable modules $A, B$, and $M$, and for some $j$, where $\Phi(j, \infty)=\mathcal{A} \cdot t^{2^{j}} \subset \mathbb{Z} / 2[t]$. Furthermore $M=\bar{T} N$. (A weaker version of this appears in [13], with the full statement appearing in [17].)

It now easily follows that, given any large enough $k$, an appropriate "subquotient" $X$ of $Y$ will satisfy $H^{*}(X)=M \otimes \Phi(k, k+2)$. This contradicts Theorem 1.1.

\section{References}

[1] J F Adams, On the non-existence of elements of Hopf invariant one, Ann. of Math. (2) 72 (1960) 20-104 MR0141119

[2] S T Ahearn, N J Kuhn, Product and other fine structure in polynomial resolutions of mapping spaces, Algebr. Geom. Topol. 2 (2002) 591-647 MR1917068

[3] G Arone, A generalization of Snaith-type filtration, Trans. Amer. Math. Soc. 351 (1999) 1123-1150 MR1638238

[4] R R Bruner, JP May, J E McClure, M Steinberger, $H_{\infty}$ ring spectra and their applications, Lecture Notes in Math. 1176, Springer, Berlin (1986) MR836132 
[5] F R Cohen, T J Lada, J P May, The homology of iterated loop spaces, Lecture Notes in Math. 533, Springer, Berlin (1976) MR0436146

[6] D Davis, moderator, Algebraic topology discussion list Available at http:// www. lehigh. edu/ dmd1/algtop.html

[7] F-X Dehon, G Gaudens, Espaces profinis et problèmes de réalisabilité, Algebr. Geom. Topol. 3 (2003) 399-433 MR1997324

[8] E E Floyd, The number of cells in a non-bounding manifold, Ann. of Math. (2) 98 (1973) 210-225 MR0334256

[9] G Gaudens, Bocksteins and the nilpotent filtration on the cohomology of spaces, from: "Proceedings of the School and Conference in Algebraic Topology", Geom. Topol. Monogr. 11, Geom. Topol. Publ., Coventry (2007) 59-79 MR2402801

[10] T G Goodwillie, Calculus. I. The first derivative of pseudoisotopy theory, $K$-Theory 4 (1990) 1-27 MR1076523

[11] T G Goodwillie, Calculus. II. Analytic functors, K-Theory 5 (1991/92) 295-332 MR1162445

[12] T G Goodwillie, Calculus. III. Taylor series, Geom. Topol. 7 (2003) 645-711 MR2026544

[13] N J Kuhn, On topologically realizing modules over the Steenrod algebra, Ann. of Math. (2) 141 (1995) 321-347 MR1324137

[14] N J Kuhn, M Slack, F Williams, Hopf constructions and higher projective planes for iterated loop spaces, Trans. Amer. Math. Soc. 347 (1995) 1201-1238 MR1282890

[15] J P May, The geometry of iterated loop spaces, Lectures Notes in Math. 271, Springer, Berlin (1972) MR0420610

[16] R J Milgram, Unstable homotopy from the stable point of view, Lecture Notes in Math. 368, Springer, Berlin (1974) MR0348740

[17] L Schwartz, À propos de la conjecture de non-réalisation due à N. Kuhn, Invent. Math. 134 (1998) 211-227 MR1646599

[18] L Schwartz, La filtration de Krull de la catégorie $\mathcal{U}$ et la cohomologie des espaces, Algebr. Geom. Topol. 1 (2001) 519-548 MR1875606

[19] V A Smirnov, Simplicial and operad methods in algebraic topology, Transl. of Math. Monogr. 198, Amer. Math. Soc. (2001) MR1811110 Translated from the Russian manuscript by G L Rybnikov

Department of Mathematics, University of Virginia

Charlottesville, VA 22904, USA

njk4x@virginia.edu

Received: 8 July 2008 Revised: 10 October 2008 